\title{
Recurrence of right lower lobe pneumonia 3 years after the first episode in an otherwise healthy 13-year-old girl
}

\author{
Antonino Capizzi, Oliviero Sacco, Michela Silvestri, Giovanni A. Rossi \\ Department of Pediatrics, Pulmonary and Allergy Disease Unit and Cystic Fibrosis Center, \\ Institute Giannina Gaslini, Genoa, Italy
}

\begin{abstract}
Recurrent pneumonia is one of the most frequent reasons for referral to paediatric chest physicians. The diagnostic work-up is dependent on whether infection repeatedly occurs in the same lung lobe, or affects multiple lobes and/or different areas in different episodes. A 13-year-old girl was admitted with a second episode of right lower lobe pneumonia. The chest x-ray demonstrated an inhomogeneous opacity, without a clearly recognizable segmental distribution. A contrast-enhanced CT scan, was therefore performed that showed a polycyclic consolidation with blood supply from a systemic artery, originated from the thoracic aorta. A diagnosis of superinfection of an intralobar sequestration was made. The patient was treated with systemic antibiotics and, four weeks later, a segmental resection of the lesion was performed. The histological evaluation of the surgical specimen confirmed the diagnosis.
\end{abstract}

\section{Introduction}

Pneumonia is a common disorder in children, also in the developed world, where its annual incidence is estimated to be 14.5 per 10,000 in children 0 to 16 years [1]. A subgroup of these children (up to 8\%) suffers from recurrent pneumonia, related to an underlying illness that

Corresponding author: Giovanni A. Rossi, Pediatric Pulmonology and Allergy Units, Cystic Fibrosis Regional Centre, IRCCS G. Gaslini, Via Gerolamo Gaslini 5,16147 Genoa, Italy. Tel. +39 01056363564 - Fax +39 0103760013. E-mail: giovannirossi@gaslini.org

Key words: Congenital malformation; pulmonary sequestration.

Funding: Supported by Ministero della Salute (Grant 5 x 1,000-2006 to Oliviero Sacco)

Conflict of interest: the authors declare that there are not potential conflict of interest, real or perceived with this manuscript.

Received for publication: 7 December 2016

Accepted for publication: 10 March 2017

(C) Copyright A. Capizzi et al., 2017

Tipografia PI-ME Editrice, Italy

Monaldi Archives for Chest Disease 2017; 87:802

doi: 10.4081/monaldi.2017.802

This article is distributed under the terms of the Creative Commons Attribution Noncommercial License (by-nc 4.0) which permits any noncommercial use, distribution, and reproduction in any medium, provided the original author(s) and source are credited. may be identified in the majority of them [2]. Pneumonias that occur in varied sites, or affect more than one lobe, suggest the presence of a more, often already known, generalized abnormality [3-5]. Examples include psychomotor retardation with feeding problems, swallow dysfunction or aspiration, congenital heart disease or severe immunodeficiencies [5]. In such patients, there is often no need to pursue another underlying etiologic cause. Conversely, infiltrates that recur in a single lobe or segment of the lung may be caused by congenital or acquired airway obstruction or by anatomic abnormalities of the lung structures. In almost all these cases there is the need to proceed further, carefully selecting the investigations to be used in each individual, taking into account the morphology and the location of the pulmonary lesion [2-5].

\section{Case report}

A 13-year-old girl was admitted to the Pulmonary and Allergy Units of the Gaslini Children Hospital for a second episode of pneumonia. She was born at 40 weeks' gestation from a regular pregnancy, with a birth weight of 2620 g. No morphologic anomalies were detected by prenatal ultrasound imaging. At 11 years of age, she was admitted to a local hospital for dry cough, fever $\left(38.5^{\circ} \mathrm{C}\right)$, right pleuritic chest pain and light respiratory distress. On the basis of the chest x-ray and blood test results (neutrophilia and elevated C-reactive protein levels), a diagnosis of lower right lobe pneumonia was made. At that time, she was treated with a third-generation cephalosporin i.v., with a positive clinical response. On admission, low-grade fever $\left(37.7^{\circ} \mathrm{C}\right)$ and normal arterial oxygen saturation in room air (98\%) were detected, associated with reduced breath sounds over the lower right field. The laboratory findings showed elevation of the C-reactive protein $(7.75 \mathrm{mg} / \mathrm{dl}$, normal range $<0.46 \mathrm{mg} / \mathrm{dl})$ and platelet counts (548,000 cells/ mmc) and the chest x-ray demonstrated an inhomogeneous paracardiac opacity in the lower right lung field, without a recognizable segmental distribution (Figure 1A). A contrast-enhanced CT scan was therefore performed and showed, in the posterior portion of the lower right lobe, a sharply circumscribed polycyclic consolidation, with an airfluid level (Figure 1 B,C). Blood supply from a systemic artery, $7 \mathrm{~mm}$ in diameter, originated from the aorta was also detected (Figure 1D, white thin arrow), better defined by a 3D reconstruction (Figure 2, black thin arrow), features consistent with a diagnosis of intralobar sequestration, complicated by superimposed infection. The patient was treated with i.v. ceftriaxone and teicoplanin. Four weeks after, a second contrast-enhanced CT scan demonstrated the regression of the polycyclic consolidation (Figure 2 A-D), with persistence of the systemic arterial blood supply (black thin arrows). A postero-lateral thoracotomy through the right sixth intercostal space was performed with a complete segmental resection of the lesion. The histological examination of the resected tissue demonstrated multiple cysts, lined with ciliated respiratory epithelium and surrounded by focal lymphoid aggregates, confirming the diagnosis [6]. The post-surgical clinical course was good with no complications. 

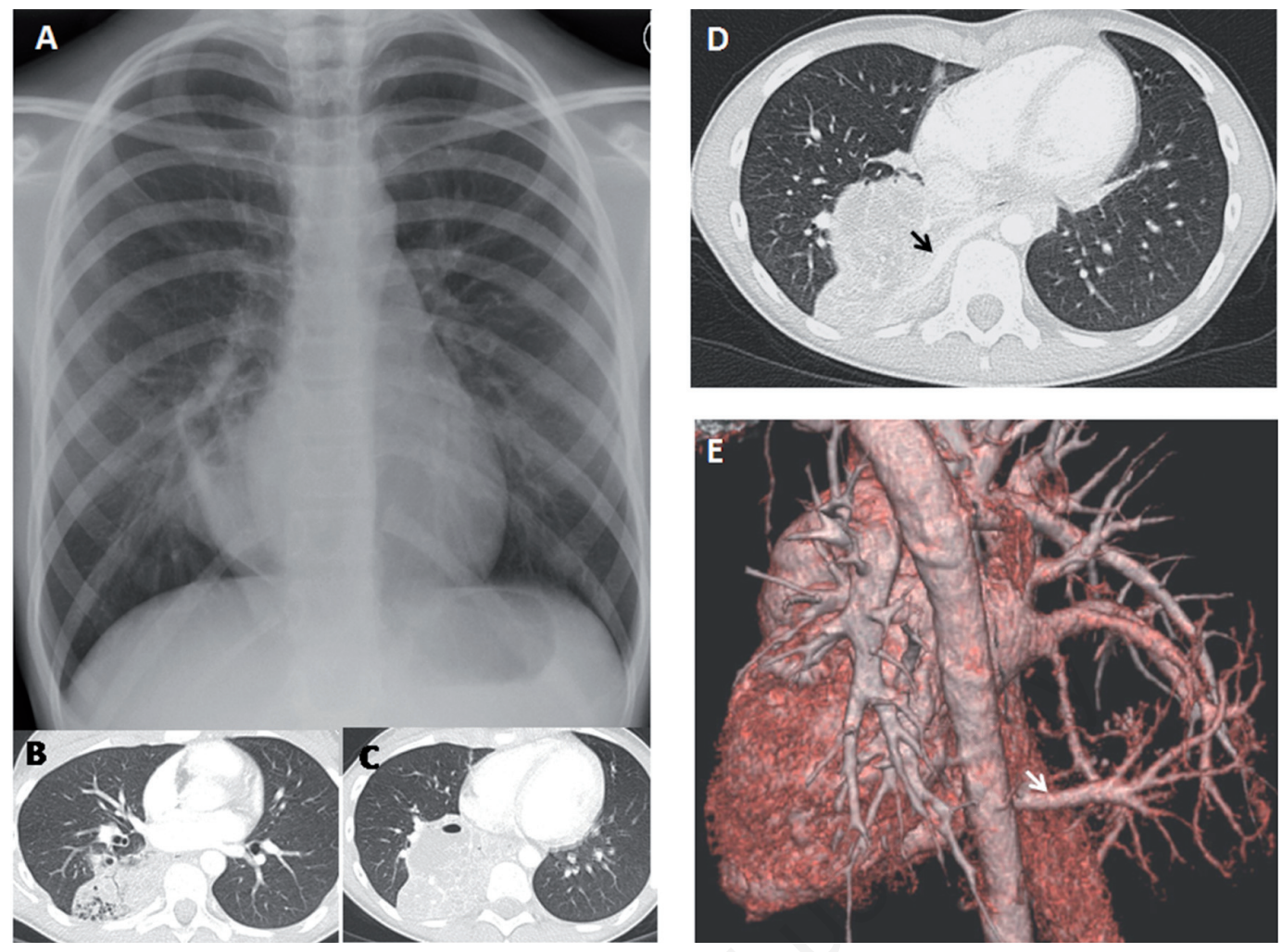

Figure 1. Radiologic evaluation of the girl at admission. A) Chest $\mathrm{x}$-ray showing inhomogeneous paracardiac opacity in the lower right lung field, without a recognizable segmental distribution. B,C) Contrast-enhanced CT scan axial views demonstrating a sharply circumscribed polycyclic consolidation, with an air-fluid level, in the posterior portion of the lower right lobe. D,E) Contrast-enhanced CT scan axial view and tridimensional reconstruction depicting the systemic artery, originated from the aorta, supplying blood to the pulmonary sequestration (black and white thin arrows).

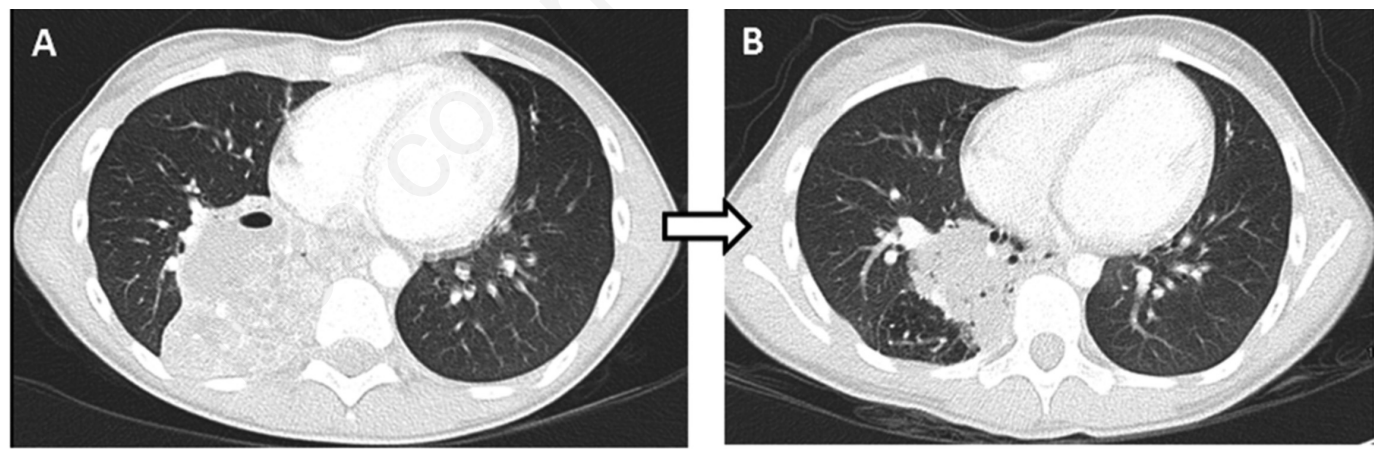

Before antibiotic treatment

After antibiotic treatment

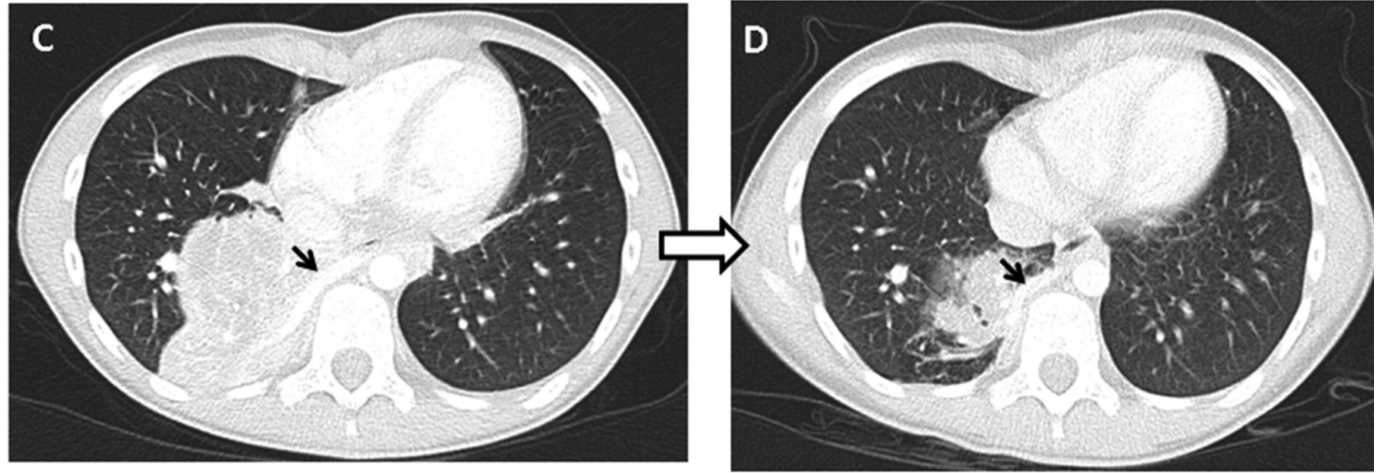

Figure 2. Contrast-enhanced CT scan axial views of the intralobar sequestration before $(A, C)$ and after $(B, D)$ antibiotic treatment. 


\section{Discussion}

Pulmonary sequestrations are a rare congenital entity accounting for 0.15 to $6.4 \%$ of all congenital pulmonary malformations. They consist in a non-functioning mass of normal lung tissue, that lacks normal communication with the tracheobronchial tree, and receives its arterial blood supply from the systemic circulation [7,8]. The most widely accepted theory suggests that pulmonary sequestrations result from formation of an accessory lung bud that develops from the ventral aspect of the primitive foregut and receives blood supply from vessels directly connected to the aorta [7-9]. Sequestrations are classified as "extralobar" or "intralobar", depending on whether the lesion is located within a normal lobe and lacks its own visceral pleura, or is located outside the normal lung and has its own visceral pleura [8]. The two varieties show different, although overlapping, clinical characteristics. The extralobar variety accounts for $25 \%$ of all sequestrations: a male to female ratio of 3 4: 1 has been reported by most authors, and the left posterior costophrenic sulcus may be affected in up to $90 \%$ of the patients. Most extralobar sequestrations present in the first 6 months of life: more than $60 \%$ of the affected infants have co-existent congenital anomalies and about $25 \%$ of them the cases in the paravertebral gutter of the posterior segment of the left lower lobe, but it is usually an isolated anomaly and no gender predominance has been recorded [8]. In addition, unless detected during antenatal ultrasounds, the intralobar sequestrations rarely cause problems before the age of 2 years. As in the case here reported, their presentation is usually in the form of chronic or recurrent pneumonia, although a small number of patients may show high output cardiac failure, haemoptysis or massive intra-thoracic bleeding [8-10]. The parenchymal changes associated with pulmonary sequestrations are variable. Many sequestrations are small and visible on a chest $\mathrm{x}$-ray only when bacterial superinfection occurs. Others may present as a single display respiratory distress or feeding difficulties shortly after birth [810]. In slightly older children, respiratory symptoms, occasionally with congestive cardiac failure, may occur [8,10-12]. The intralobar variety is also located in $60 \%$ of homogeneous opacity or a well-defined triangular shaped or a cystic mass, or, less commonly, a cystic mass eventually with air fluid levels, caused by a bronchial communication, in $26 \%$ of cases $[12,13]$. The most common complication of intralobar sequestrations is infection by bacteria migrating through the pores of Kohn or by other routes, if the sequestration is incomplete. The standard treatment is resection of the segment or lobe that contains the sequestered tissue $[14,15]$. Resection of intralobar sequestrations almost always involves a lobectomy, whilst segmental resections are usually only possible in lesions detected before the onset of infection. Other options are ligation or embolization of the vascular pedicle. However, incomplete embolization and incomplete or null regression of the sequestered tissue are reported in $25-47 \%$ of pediatric cases $[12,16]$. Some authors advocate conservative antibiotic treatment with surgical intervention only in cases of repeated infections; however, this strategy is rarely used because of the potential of bleeding complications from repeated infections [15].

\section{Conclusions}

In our patient, the presence of a pulmonary sequestration was suspected not only on the basis of the history of a previous pneumonia affecting the same lung lobe, but mainly on the radiographic characteristics of the inhomogeneous opacity, lacking recognizable segmental distribution. The suspicion was confirmed by a contrast-enhanced CT scan, the approach that best demonstrates the parenchymal abnormalities associated with pulmonary sequestration [8]. In evaluating children with recurrent episodes of pneumonia, physicians must carefully evaluate of each episode, with a complete review of all available chest radiographs, when present. An early diagnosis of a pulmonary malformation may allow performing, as in our patient, a segmental resection, preserving the residual healthy lung parenchyma.

\section{References}

1. Harris M, Clark J, Coote N, et al. British Thoracic Society guidelines for the management of community acquired pneumonia in children: update 2011. Thorax 2011;66 Suppl 2:ii1.

2. Owayed AF, Campbell DM, Wang EE. Underlying causes of recurrent pneumonia in children. Arch Pediatr Adolesc Med. 2000; 154:190-4.

3. Panitch HB. Evaluation of recurrent pneumonia. Pediatr Infect Dis J 2005;24:265-6.

4. Vaughan D, Katkin JP. Chronic and recurrent pneumonia in children. Semin Respir Inf 2002;17:73-84.

5. Brand PL, Hoving MF, de Groot EP. Evaluating the child with recurrent lower respiratory tract infections. Paediatr Respir Rev 2012; 13:135-8.

6. Fowler DJ, Gould SJ. The pathology of congenital lung lesions. Semin Pediatr Surg 2015;24:176-82.

7. Sade RM, Clouse M, Ellis FH. The spectrum of pulmonary sequestration. Ann Thorac Surg 1974;18:644-58.

8. Corbett HJ, Humphrey GM. Pulmonary sequestration. Paediatr Respir Rev 2004;5:59-68.

9. Stocker JT. Sequestrations of the lung. Semin Diagn Pathol 1986;3:106-21.

10. Savic B, Birtel FJ, Tholen W, et al. Lung sequestration: report of seven cases and review of 540 published cases. Thorax 1979;34: 96-101.

11. Thilenius OG, Ruschhaupt DG, Replogle RL, et al. Spectrum of pulmonary sequestration: association with anomalous pulmonary venous drainage in infants. Pediatr Cardiol 1983;4:97-103.

12. Tokel K, Boyvat F, Varan B. Coil embolization of pulmonary sequestration in two infants: a safe alternative to surgery. AJR Am J Roentgenol 2000;175:993-5.

13. John PR, Beasley SW, Mayne V. Pulmonary sequestration and related congenital disorders a clinico-radiological review of 41 cases. Pediatr Radiol 1989;20:4-9.

14. Kestenholz PB, Schneiter D, Hillinger S. Thoracoscopic treatment of pulmonary sequestration. Eur. J. Cardiothorac. Surg 2006;29: $815-8$.

15. Shibli M, Connery C, Shapiro JM. Intralobar and extralobar bronchopulmonary sequestration complicated by Nocardia asteroides infection. South Med J 2003;96:78-80.

16. Chien KJ, Huang TC, Lin CC, et al. Early and late outcomes of coil embolization of pulmonary sequestration in children. Circ J 2009; 73:938-42. 